\title{
HÉRNIA DIAFRAGMÁTICA TRAUMÁTICA
}

\author{
TRAUMATIC DIAPHRAGMATIC HERNIA
}

Gerson Alves Pereira Júnior, TCBC-SP ${ }^{1}$

\section{INTRODUÇÃO}

A ruptura diafragmática foi descrita inicialmente por Sennertus, em 1541, quando relatou a autópsia de um caso de herniação e estrangulamento do estômago através de um defeito diafragmático devido a uma antiga (sete meses) lesão de guerra ${ }^{1}$.

Ambroise Paré, em 1579, relatou dois casos de pacientes com hérnia diafragmática diagnosticados na autópsia, sendo que a causa de morte foi uma obstrução jejunal e a outra colônica, secundárias à herniação transtorácica, tendo descrito as três fases de apresentação: fase aguda, fase de intervalo (sintomas vagos) e fase crônica (obstrução ou estrangulamento) ${ }^{1-4}$.

Bowditch publicou, em 1853, os primeiros casos diagnosticados pré-óbito de hérnia diafragmática e estabeleceu cinco critérios para o seu diagnóstico no exame físico: imobilidade do hemitorax, deslocamento da macicez da área cardíaca para a direita, ruídos hidroaéreos audíveis no tórax, ausência de murmúrio vesicular e timpanismo no tórax ${ }^{1,2,4}$.

Riolfi, em 1886, realizou a primeira sutura de um ferimento por arma branca do diafragma, obtendo sucesso ${ }^{5}$.

\section{PATOGENIA}

Entre as cavidades abdominal e torácica, há um gradiente de pressão que varia normalmente entre 7 e $22 \mathrm{cmH}_{2} \mathrm{O}$, podendo atingir $100 \mathrm{cmH}_{2} \mathrm{O}$ durante a inspiração forçada máxima, quando a pressão interpleural é mais negativa ${ }^{1,3.5,6}$.

No trauma abdominal contuso grave, há um aumento de dez vezes na pressão abdominal, transmitindo subitamente a energia cinética através das cúpulas frênicas ${ }^{6}$. Qualquer porção do diafragma pode ser lesada, entretanto, a maioria das lacerações ocorre em direção radial na área póstero-lateral do diafragma esquerdo, que corresponde a um ponto embriológico de fraqueza. ${ }^{1,6}$. O centro tendíneo do diafragma, por ser inextensível, também é sede frequiente de lesões ${ }^{7,8}$.

\section{EPIDEMIOLOGIA}

A ruptura diafragmática ocorre em $0,8 \%$ dos pacientes vítimas de acidentes automobilísticos e em cerca de $3 \%$ a 5\% dos casos de traumatismo abdominal fechado ${ }^{6,8}$.

Ocorre também após trauma penetrante (cerca de $13 \%$ a $19 \%$ projétil lesam o diafragma $)^{5}$, tanto por arma de fogo $(46 \%)$ como por arma branca (15\%), sendo que no primeiro caso, como invariavelmente estes pacientes são submetidos à cirurgia, o diagnóstico da lesão é realizado no período intra-operatório. No caso de ferimento por arma branca, a crescente conduta de seleção dos pacientes para indicação cirúrgica, com base na exploração local do ferimentos na parede abdominal anterior, tentando certificar-se da integridade ou da violação do peritônio e, na tomografia computadorizada do abdome com triplo contraste (via oral, endovenoso e retal) nos ferimentos do dorso ${ }^{9}$, contribuiu para uma redução de três vezes no número de laparotomias desnecessárias ${ }^{10}$, ao contrário da exploração peritoneal de rotina, antigamente realizada, principalmente em ferimentos da transição toracoabdominal.

O número de hérnias diafragmáticas na sua forma crônica tem aumentado, pois lesões diafragmáticas isoladas, que chegam a ocorrer em $5 \%$ a $19 \%$ dos ferimentos toracoabdominais, particularmente à esquerda, permanecem assintomáticas na ausência de sangramento ou de lesão de víscera oca ${ }^{10}$, diagnosticados ocasionalmente num exame radiológico do tórax ou quando ocorrem complicações, como a obstrução intestinal ou o estrangulamento de vísceras ocas herniadas ${ }^{11,12}$.

Os ferimentos penetrantes permanecem como a principal causa de lesões diafragmáticas despercebidas, pois tais lesões, com extensão média de 2 a $4 \mathrm{~cm}$, são menores do que as do trauma contuso, que variam de 5 a $15 \mathrm{~cm}$ de extensão $\mathrm{O}^{2,5,7,11}$. A maioria está localizada perifericamente, próximo ao sítio de entrada na parede torácica. ${ }^{5}$. Os pequenos ferimentos são os que mais comumente apresentam-se com estrangulamento e obstrução do que os grandes ${ }^{13}$.

1. Médico Assistente da Unidade de Emergências do Hospital das Clínicas da Faculdade de Medicina de Ribeirão Preto (FMRP) da Universidade de São Paulo (USP).

Recebido em 22/09/1999

Aceito para publicação em 31/10/2000

Trabalho realizado na Unidade de Emergências do Hospital das Clínicas da Faculdade de Medicina de Ribeirão Preto da Universidade de São Paulo. 
A hérnia diafragmática é definida como a evisceração transdiafragmática de conteúdo abdominal no torax ${ }^{3}$. Os órgãos abdominais mais frequentemente herniados através do defeito diafragmático são o estômago, omento, cólons e fígado ${ }^{5}$. Muitas lesões, especialmente no trauma penetrante, não podem ser qualificadas como verdadeiras hérnias diafragmáticas, já que o ferimento é pequeno, não permitindo a herniação de vísceras para o tórax ${ }^{3,14}$.

A hérnia diafragmática, no trauma contuso, ocorre com igual frequência à direita e à esquerda em levantamentos realizados em autópsias ${ }^{7,8}$, porém devido a maior energia transmitida no trauma para que ocorra a lesão do diafragma direito, com maior gravidade das lesões associadas, há maior mortalidade pré-hospitalar nestes casos ${ }^{7}$, sendo mais freqüente o diagnóstico hospitalar de hérnias diafragmáticas à esquerda $(72 \% \mathrm{a} 90 \%)^{6,8,15,16}$. Entretanto, com a melhora do serviço de resgate pré-hospitalar, há uma tendência de aumento do diagnóstico do número de casos de hérnia diafragmática direita ${ }^{3,7,8}$.

Nos traumas penetrantes por arma branca, o lado esquerdo é o mais freqüentemente lesado (66\% a 77\%), visto que a maioria dos agressores são destros ${ }^{2,5,6}$, enquanto que há igual distribuição nos ferimentos por arma de fogo ${ }^{5,6}$. Também deve-se levar em conta que o fígado não é capaz de herniar por pequenas lesões do lado direito ${ }^{1,10}$. Todos estes aspectos comprovam a maior probabilidade de hérnias diafragmáticas crônicas do lado esquerdo.

O impacto lateral no trauma contuso tem três vezes mais chance de resultar em ruptura diafragmática do que o impacto frontal, com tendência a ruptura ipsilateral do lado do impacto ${ }^{7}$. É rara a presença de hérnia diafragmática direita não associada a acidentes automobilísticos ${ }^{7}$. Em $2 \%$ a $5 \%$ dos casos a lesão diafragmática pode ser bilateral ${ }^{1}$.

A hérnia diafragmática deve ser diferenciada da eventração diafragmática, que é uma elevação permanente da musculatura frênica devido a aplasia, paralisia ou atrofia, porém o diafragma mantém sua continuidade e inserção na margem costal, raramente produzindo sintomas e não requerendo tratamento ${ }^{17,18}$. Em pacientes sintomáticos, a plicatura do diafragma pode oferecer alívio dos sintomas ${ }^{18}$.

\section{CLASSIFICAÇÃO}

A história natural das hérnias diafragmáticas é descrita em três fases:

1) fase aguda: quando o paciente apresenta-se com a lesão, que pode ter expressão clínica variável, dependendo do tipo de trauma e da própria extensão da lesão. Se não diagnosticada e reparada cirurgicamente, evoluirá para as fases seguintes:

2) fase intermediária ou latente: é uma fase de encarceramento das vísceras abdominais no tórax pela constante pressão pleural negativa, variando conforme o diâmetro da lesão diafragmática, que geralmente produz sintomatologia vaga, como dor epigástrica, náusea, vômitos e episódios de obstrução intestinal;

3) fase crônica: é a fase onde ocorrem as complicações, principalmente obstrução e estrangulamento intestinal. Há relatos de hérnias diagnosticadas de 28 dias até 28 $\operatorname{anos}^{2,19}$. Cerca de $85 \%$ dos casos são diagnosticados dentro de três anos após o trauma ${ }^{5,13}$.

A classificação internacional mais utilizada para descrição da lesão é a do Organ Injury Scaling ${ }^{20}$ da Associação Americana de Cirurgia do Trauma, a ser utilizada no período intra-operatório (ver Tabela 1).

\section{DIAGNÓSTICO}

As lesões diafragmáticas representam um desafio diagnóstico para os cirurgiões pela variabilidade de sua apresentação clínica e pelo emprego de vários métodos diagnósticos disponíveis. ${ }^{5}$

O diagnóstico clínico é baseado no mecanismo de trauma, tanto para os ferimentos contusos quanto para os penetrantes, sendo necessário um alto índice de suspeita ${ }^{15,21}$. A sintomatologia e os achados no exame físico podem ser torácicos ou abdominais 5 .

O exame físico, raramente, fornece o diagnóstico, uma vez que estes pacientes chegam em estado grave na sala de admissão, não havendo tempo hábil para um exa-

Tabela 1

Classificação da Organ Injury Scaling para Trauma Diafragmático

\begin{tabular}{l|lc}
\hline Grau & \multicolumn{1}{|c}{ Descrição da Lesão } & Abreviated Injury Scale (AIS) \\
\hline I & Contusão & 3 \\
II & Laceração $<=2 \mathrm{~cm}$ & 3 \\
III & Laceração de 2 a $10 \mathrm{~cm}$ & 3 \\
IV & Laceração $>10 \mathrm{~cm}$ com perda de tecido $<=25 \mathrm{~cm}^{2}$ & 3 \\
V & Laceração com perda tecidual maior que $25 \mathrm{~cm}^{2}$ & 3
\end{tabular}


me minucioso. Entre $38 \%$ e $59 \%$ dos pacientes têm achados físicos devido às lesões associadas, como hemo/pneumotórax ou peritonismo ${ }^{5}$ O sinal de Gibson, com o abdome escavado mostrando assimetria dos hipocôndrios é pouco encontrado ${ }^{8}$. A dificuldade de passagem do cateter nasogástrico na junção esofagogástrica é altamente sugestiva de lesão diafragmática com herniação do estômago para o tórax ${ }^{8}$.

A região de transição toracoabdominal é a área compreendida anterior e superiormente pelos mamilos (4으 espaço intercostal), posterior e superiormente, pela ponta da escápula (7o espaço intercostal) e, inferiormente, pelo rebordo costal. Esta área contém o diafragma durante a sua excursão nos ciclos respiratórios. Pacientes assintomáticos com ferimentos penetrantes nesta área devem ser investigados imediatamente para excluir lesão diafragmática ${ }^{5}$. A exploração local dos ferimentos não é recomendada pelo risco de induzir a um pneumotórax ${ }^{5,22}$.

A radiografia simples de tórax, geralmente revela as hérnias diafragmáticas agudas, mostrando um padrão de gás anormal acima do hemidiafragma com elevação e irregularidade, principalmente em casos de trauma contuso ${ }^{2,23}$. Nos casos de trauma penetrante é frequente o exame ser normal em $36 \%$ a $67 \%$ dos $\operatorname{casos}^{2,5,23}$ (Figura 1). É importante a realização da radiografia de tórax em perfil, pela grande incidência de lesões diafragmáticas posteriores ${ }^{8}$. Outras características radiológicas em casos de hérnias diafragmáticas, menos freqüentemente encontrados, são: falha da radiografia de tórax retornar ao normal durante o tratamento com drenagem pleural e o hemotórax persistente ${ }^{2,23}$.

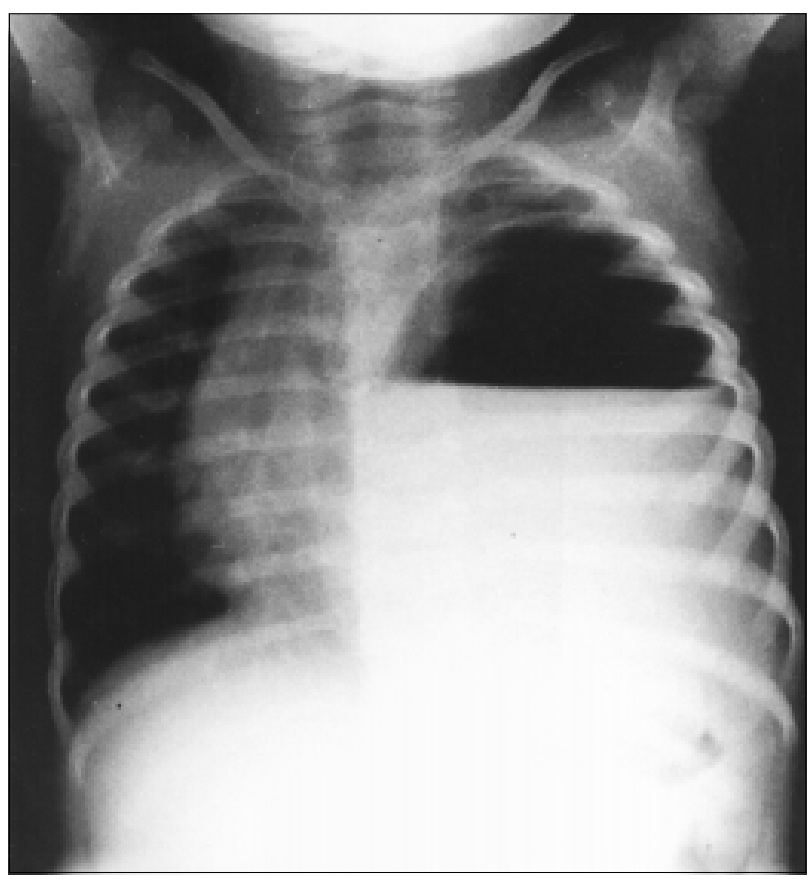

Figura 1 - Radiografia em posição ântero-posterior, evidenciando nível líquido em hemitorax esquerdo, desviando o mediastino para o lado contralateral, compatível com hérnia diafragmática.
O diagnóstico pode ser confirmado com a passagem de uma sonda nasogástrica ou mesmo com o uso de contraste radiológico, de preferência, iodado, dado por via oral ou por cateter (Figura 2). A colocação do paciente em posição de Trendelenburg ou o aumento da pressão intra-abdominal durante a realização da radiografia de tórax são úteis para facilitar o diagnóstico, porém têm sido pouco utilizados ${ }^{2}$.

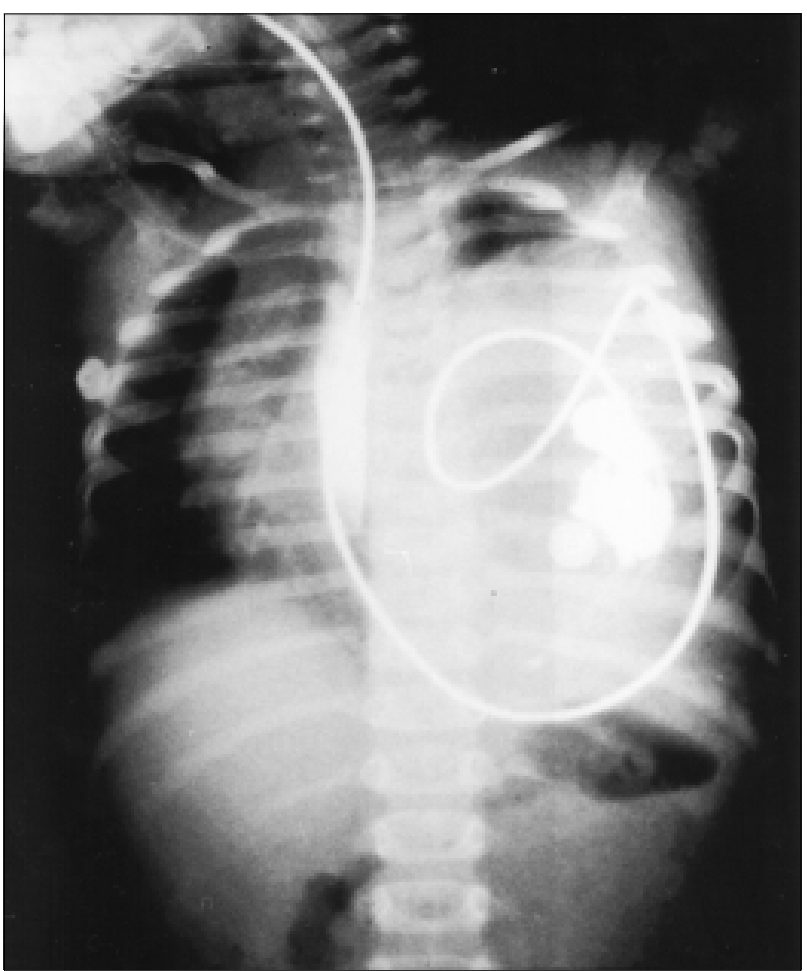

Figura 2 - Radiografia de tórax em posição ântero-posterior, após a passagem de cateter nasogástrica e injeção de contraste, mostrando a presença do estômago dentro do hemitorax esquerdo, confirmando o diagnóstico de hérnia diafragmática.

Outros métodos descritos para diagnóstico são: radioscopia do hemidiafragma, pneumoperitôneo diagnóstico $^{24}$, pleurografia ${ }^{25}$, peritoneografia ${ }^{25}$, cintilografia com injeção intraperitoneal de tecnécio ${ }^{26}$, ultrassonografia computadorizada ${ }^{27}$, tomografia de tórax ${ }^{28}$, ressonância nuclear magnética, toracoscopia, laparoscopia, lavado peritoneal, exploração digital na drenagem pleural e na laparotomia $^{2,6,23}$. Na ultra-sonografia, o diafragma não é bem visualizado devido a sombra dos gases ao redor, sendo que apenas a superfície posterior do diafragma esquerdo e a cúpula do direito podem ser visualizadas com certeza ${ }^{27}$.

Há algumas apresentações pouco usuais da hérnia diafragmática traumática, podendo ter localização intrapericárdica, quando a ecocardiografia e a ultra-sonografia auxiliam no diagnóstico ${ }^{29,30} \mathrm{ou}$, ainda, apresentando-se como hérnia intercostal ${ }^{31}$.

Como a presença de hérnia diafragmática pode determinar sinais e sintomas que sugiram a necessidade de 
drenagem torácica, é imperativo que se faça o diagnóstico correto, pelo risco de drenagem iatrogênica da víscera herniadas ${ }^{8}$. Para evitar tal complicação, o Advanced Trauma Life Support (ATLS) preconiza a drenagem pleural na linha mamilar entre a linha axilar média e anterior, sendo necessária a exploração do oríficio com o dedo indicador, antes da introdução do dreno pleural ${ }^{32}$.

A radiografia de tórax, usualmente, fornece o diagnóstico, mostrando mais comumente as seguintes anormalidades: elevação com irregularidade do hemidiafragma, padrão anormal de gás acima do diafragma com alças distendidas e cateter nasogástrica dentro do hemitórax ${ }^{2,23}$.

$\mathrm{O}$ uso de contraste radiológico pode ser feito tanto para diagnóstico de hérnias agudas quanto crônicas para documentar a posição anormal do estômago ou cólon no hemitorax esquerdo, usando-se de preferência contrastes iodados, pelo risco da presença de perfuração de vísceras ocas e peritonite química intensa no extravasamento de bário 2,23 .

O lavado peritoneal diagnóstico realizado em paciente com um hemitorax drenado com saída de líquido do lavado pelo dreno, confirma o diagnóstico de hérnia diafragmática ${ }^{8,33}$, porém a taxa de lavado peritoneal falso-negativo em casos de hérnia diafragmática é relatada entre $25 \%$ e $40 \%$, tendo como possíveis causas a hemorragia contida na retrocavidade dos epíploons por lesão diafragmática posterior esquerda, hemorragia seletiva para dentro da cavidade torácica ou tamponamento imediato do sangramento pela herniação de vísceras ${ }^{1,7,8,10}$. Na maioria dos casos, a positividade do lavado peritoneal se deve a presença de lesões intra-abdominais associadas ${ }^{8}$.

Atualmente, o lavado peritoneal tem sido utilizado para excluir lesões intra-abdominais em pacientes assintomáticos com ferimentos penetrantes por arma branca em dorso e flancos, bem como em ferimentos penetrantes por arma branca toracoabdominais (entre os mamilos, anteriormente, ou a ponta das escápulas, posteriormente, e as margens costais) e ferimentos tangenciais por arma de fogo, sendo usado o limite de 5.000 hemácias $/ \mathrm{mm}^{3}$ como uma contagem positiva, em vez de 100.000 hemácias $/ \mathrm{mm}^{3}$, como é de rotina, pelo risco de lesões despercebidas. Assim, os centros que adotam o lavado peritoneal para a avaliação de ferimentos toracoabdominais terão que optar entre um aumento do número de laparotomias negativas (se a contagem de 5.000 hemácias $/ \mathrm{mm}^{3}$ é usada) contra um ocasional pequeno defeito diafragmático despercebido (se a contagem de 100.000 hemácias $/ \mathrm{mm}^{3}$ é utilizada) ${ }^{2}$.

Num estudo prospectivo realizado em pacientes com ferimentos toracoabdominais penetrantes, assintomáticos e estáveis hemodinamicamente, a laparotomia exploradora foi indicada independentemente dos resultados obtidos no lavado peritoneal diagnóstico e encontrou-se uma acurácia de 94,6\% do lavado peritoneal diagnóstico com contagem de hemácias de $10.000 / \mathrm{mm}^{3}$, com apenas $3,5 \%$ de falso-negativos (lesão despercebida), diminuindo o número de laparotomias não-terapêuticas para $12,5 \%{ }^{33}$.

Uma outra abordagem que pode ser utilizada em casos de ferimentos penetrantes toracoabdominais posteriores, em que é difícil a exploração local do ferimento em virtude da densa musculatura da região, é a realização de tomografia computadorizada do abdome com triplo contraste (via oral, endovenosa e retal) ${ }^{9}$.

Nas hérnias diafragmáticas crônicas, a apresentação clínica é variável. Pode apresentar-se com sinais e sintomas de obstrução intestinal e, se estrangulada, com sinais de isquemia intestinal e sepse 5 . Outras vezes, o diagnóstico é feito através de uma radiografia simples de tórax por qualquer outra causa, sendo um achado incidental. A apresentação clínica pode não ocorrer até a gravidez ou trabalho de parto, quando o aumento da pressão intra-abdominal predispõe à herniação ${ }^{34}$.

A presença de hérnia diafragmática crônica é fortemente sugerida no diagnóstico diferencial de portadores de obstrução intestinal nos seguintes grupos de pacientes ${ }^{35}$ :

- aqueles com história de trauma toracoabdominal penetrante;

- aqueles onde se constata a presença de derrame pleural associado ao quadro de obstrução intestinal;

- aqueles sem operação prévia ou hérnias da parede abdominal;

- nos pacientes jovens com obstrução colônica.

A laparotomia é, provavelmente, o método mais acurado para se diagnosticar a lesão diafragmática, porém, se realizada de rotina em pacientes com estabilidade hemodinâmica, na presença de ferimentos toracoabdominais penetrantes, a taxa de laparotomias não-terapêuticas pode chegar a $78 \%$, acarretando morbidade de $10 \%$ a $19 \%$, com complicações que variam desde a atelectasia e pneumonia até deiscência de ferida cirúrgica e obstrução intestinal $^{5}$.

Em casos de toracotomia ou laparotomia de urgência, tanto em casos de trauma contuso e, principalmente, de trauma penetrante, o cirurgião deve verificar a integridade das cúpulas frênicas como um procedimento de rotina no inventário da cavidade abdominal.

Para se evitar a morbidade associada a laparotomias não-terapêuticas, têm-se investigado a utilização da videocirurgia através de laparoscopia ${ }^{36}$ e toracoscopia ${ }^{37}$.

\section{LESÕES ASSOCIADAS}

O reconhecimento da ruptura diafragmática é importante pela frequência e gravidade das lesões associadas ${ }^{38}$.

Devido à grande energia cinética necessária para produzir lesão diafragmática em traumas contusos, é muito freqüente a presença de lesões associadas $(72 \% \text { a } 95 \%)^{3,6,38}$, principalmente de baço e fígado em até $25 \%$ (no tratamento conservador não-operatório destes órgãos, a lesão diafragmática pode passar despercebida) ${ }^{8}$, mas também de traumatismo cranioencefálico, fraturas de bacia $(40 \%$ a $55 \%)$ e dos membros ${ }^{3,6,8,16,38}$. A associação entre lesão diafragmática e fratura pélvica envolve a força maciça necessária para romper o anel pélvico com o súbito e drástico aumento na pressão intra-abdominal, que é transmitida às cúpulas frênicas, causando ou contribuindo para a ruptura do diafragma ${ }^{16}$. 
Em hérnias diafragmáticas do lado direito é maior o número e a gravidade das lesões associadas responsáveis por muitas das complicações. Em cerca de 3\% a 10\% dos casos, ocorre lesão da aorta torácica, particularmente em lesões do lado direito ${ }^{7}$.

Nos casos de ferimentos penetrantes, cerca de $66 \%$ dos pacientes com trauma diafragmático têm lesões associadas, mais comumente do pulmão e da pleura, resultando em hemotórax ou pneumotórax ${ }^{5}$. No abdome, os órgãos mais lesados são o baço (24\% a 36\%), o fígado (23\% a $29 \%)$ e o cólon $(17 \%)^{5}$.

\section{TRATAMENTO}

Uma vez realizado o diagnóstico de hérnia diafragmática aguda ou crônica no pré-operatório, com base em exames radiológicos ou no intra-operatório, em casos duvidosos ou com indicação cirúrgica de urgência, procedese ao tratamento cirúrgico com sutura do diafragma.

O diafragma deve sempre ser suturado, pois mesmo pequenos ferimentos, ainda que tamponados por epíploon, não têm cicatrização espontânea devido ao gradiente pressórico entre as cavidades abdominal e torácica, além da movimentação contínua do diafragma no ciclo respiratório e do risco de estrangulamento de vísceras intra-abdominais no tórax ${ }^{4,5}$.

A primeira decisão a ser tomada nos casos em que é realizado o diagnóstico pré-operatório é quanto à incisão utilizada para acesso cirúrgico. Na fase aguda, devido a grande porcentagem de lesões intra-abdominais associadas, a maioria dos cirurgiões tendem a optar pela laparotomia mediana ${ }^{2,3,6,15}$, enquanto pequena parte deles opta, inicialmente, pela toracotomia. Muitas vezes, é necessário lançar mão da ampliação da incisão (toracofrenolaparotomias). Pela maior morbidade desta incisão combinada ${ }^{39}$, de preferência, utilizamos incisões em separado no tórax e abdome, para melhor abordagem em casos de lesões complexas, particularmente em lesões do lado direito ${ }^{20}$, em que algumas revisões da literatura mostram que em até 50\% dos casos é necessária uma toracotomia complementar ${ }^{3,6}$.

No caso de hérnias diafragmáticas crônicas (> 2 semanas), a tendência geral é que se aborde a lesão através de toracotomia ${ }^{1,3}$, pela maior facilidade de redução do conteúdo herniado sob visão direta, além da lise de aderências, prováveis nesta fase $\mathrm{f}^{6,15}$.

Uma vez localizado o ferimento diafragmático, no período intra-operatório, as bordas da lesão devem ser presas com pinças de Allis longas e fracionadas em direção ao cirurgião. Inicialmente, procede-se à redução do conteúdo herniado e, para tanto, é de grande auxílio a passagem de cateter nasogástrico para descompressão do estômago herniado ou mesmo a ampliação do ferimento (extensão da frenotomia $)^{2}$, preservando-se a inervação frênica do diafragma. Normalmente, esta manobra é realizada sem dificuldades, já que não há saco herniário e não houve tempo para a formação de aderências. No caso de hérnias crônicas, a redução também é feita sem muita dificuldade, porém deve-se estar atento para aderências que podem lacerar vísceras ocas ou mesmo a cápsula de órgãos parenquimatosos, acarretando sangramentos.
Em todos os casos de lesão diafragmática, a drenagem pleural, se não realizada previamente, como é freqüente, já que muitas lesões diafragmáticas não são suspeitadas na sala de admissão, deve ser realizada no período intraoperatório, de preferência sob visão direta ou colocandose os dedos através do ferimento diafragmático pela via abdominal, para melhor posicionamento do dreno ${ }^{8}$. A drenagem pleural deve ser realizada antes do fechamento definitivo do diafragma. Se a drenagem pleural não for realizada, mesmo em lesões pequenas, o paciente evoluirá com pneumotórax, hidrotórax ou hidropneumotórax no pósoperatório, necessitando de drenagem, o que posterga a internação, com maiores riscos de aumento da morbidade.

$\mathrm{Na}$ presença de lesões de vísceras ocas associadas, principalmente nos extravasamentos colônicos, devido à maior carga de bactérias, deve se proceder à drenagem pleural logo após o controle da hemostasia e sutura das perfurações de vísceras ocas, lavando-se, exaustivamente, tanto a cavidade abdominal, quanto a torácica, permitindo o escoamento do líquido de lavagem pelo dreno pleural, tentando-se minimizar o risco de empiema pleural, uma temida complicação de díficil e prolongado tratamento pós-operatório. Alguns autores advogam a realização de toracotomia ântero-lateral para se visualizar toda a cavidade pleural e permitir a evacuação de todo o material contaminante, através de lavagem copiosa com solução salina e a colocação de dois drenos pleurais ${ }^{16}$.

A literatura médica não mostra diferenças de resultados quanto ao fechamento das lesões diafragmáticas em termos de suturas contínuas ou com pontos separados, recomendando, apenas, que seja utilizado fio inabsorvível (polipropileno). Raras vezes, principalmente, em hérnias diafragmáticas crônicas, mas também nas hérnias diafragmáticas agudas nos graus III e IV da Organ Injury Scaling $^{20}$, há perda de substância ou mesmo atrofia (nas lesões crônicas) da musculatura do diafragma, não sendo possível a sutura primária da lesão, tendo-se, então, que lançar mão de próteses, como Marlex, Dacron ou dura-máter ${ }^{14,40,41}$. Outras vezes, há uma desinserção costal da musculatura do diafragma, que deve ser novamente suturada na parede torácica. Ainda pode ocorrer a lesão do diafragma no hiato esofágico, que deve ser reconstruído, verificando-se e mantendo-se a integridade dos nervos vagos.

Na presença de destruição diafragmática por ferimento de arma de fogo toracoabdominal, podemos liberar todo o diafragma de suas inserções anterior, lateral e posterior, e translocar para uma posição acima do defeito da parede torácica, convertendo o defeito, funcionalmente, como defeito da parede abdominal. O diafragma é, então, ressuturado em um espaço intercostal mais alto ${ }^{42}$.

Após completar-se o reparo diafragmático, a integridade da linha de sutura deve ser testada pelo aumento da pressão intratorácica através do aumento do volume corrente administrado e avaliando-se a movimentação diafragmática ${ }^{16}$.

Atualmente, tem-se feito o diagnóstico e também o tratamento das lesões diafragmáticas através da videocirurgia, tanto por laparoscopia ${ }^{43,44}$, quanto por toracoscopia ${ }^{4}$. 
A acurácia da laparoscopia é excelente para hemoperitônio causado pela lesão de órgãos parenquimatosos e por lacerações do diafragma ${ }^{16}$.

Há vários argumentos em favor do uso da toracoscopia como procedimento de escolha: (1) todas as lesões diafragmáticas requerem a colocação de dreno pleural e a toracoscopia pode ser realizada através da mesma incisão, (2) permite a avaliação de outras lesões intratorácicas, (3) pode ser usada durante a abordagem diagnóstica inicial para efetivamente evacuar o hemotórax, o que evita o risco de hemotórax retido, que requer decorticação por toracotomia ou toracoscopia ${ }^{4}$.

\section{COMPLICAÇÕES}

A mais comum complicação pós-traumática do diafragma é a lesão despercebida com hérnia crônica. Pode ocorrer tanto por um aumento da frequência de lesões despercebidas, quanto por uma deiscência de uma lesão corrigida previamente ${ }^{12}$. Para minimizar tal complicação é necessário um alto índice de suspeita da presença de lesão diafragmática e o adequado reparo da lesão ${ }^{12}$.

A lesão dos ramos periféricos do nervo frênico no diafragma pode produzir denervação regional, resultando em atrofia e perda de função da região correspondente. Quando possível, deve-se evitar tais lesões durante o reparo das hérnias pós-traumáticas agudas ou crônicas ${ }^{12}$.

Anatomicamente, os nervos frênicos dividem-se no diafragma ou $1 \mathrm{a} 2 \mathrm{~cm}$ acima dele. Cada ramo divide-se em quatro ramos principais: anterior, ântero-lateral, pósterolateral e posterior. Deve-se evitar a realização de incisões radiais no diafragma para não lesar estes ramos, sendo mais aconselhado o uso de incisões circunferenciais na periferia do mesmo ${ }^{45,46}$. As incisões no centro tendíneo do diafragma localizadas medialmente à entrada do nervo frênico, bem como àquelas laterais ou incisões transversas, a partir da linha axilar média em direção medial, não causam paralisia diafragmática ${ }^{45,46}$ (Figura 3).

Deve-se estar alerta para a presença de pneumotorax durante a laparotomia exploradora, que pode manifestarse por instabilidade hemodinâmica e respiratória com diminuição da complacência pulmonar, manifestada através de elevado pico de pressão intra-traqueal, visualizada pelo anestesista durante a ventilação mecânica ${ }^{12}$. Na avaliação laparoscópica do trauma abdominal, a insuflação de ar no abdome pode produzir um pneumotorax hipertensivo através do orifício no diafragma, necessitando de drenagem pleural imediata ${ }^{12,41}$.

O empiema pleural, geralmente, ocorre quando há perfuração de vísceras ocas abdominais, particularmente na presença de hemotórax associado ${ }^{12}$.

\section{MORBIDADE E MORTALIDADE}

A mortalidade após ruptura diafragmática em casos de trauma contuso varia entre $8 \%$ e $50 \%$ nas diferentes séries, sendo a morte atribuída geralmente a lesões neurológicas, ortopédicas e intra-abdominais associadas ${ }^{1,8}$. A mortalidade em casos de hérnias diafragmáticas direitas chega a ser maior que 50\%, sendo atribuída à grande energia necessária para provocar tal lesão ${ }^{5}$.

No caso de estrangulamento de vísceras intra-abdominais herniadas, a mortalidade aumenta dramaticamente ${ }^{1,11}$, podendo chegar a $88 \%{ }^{13}$.

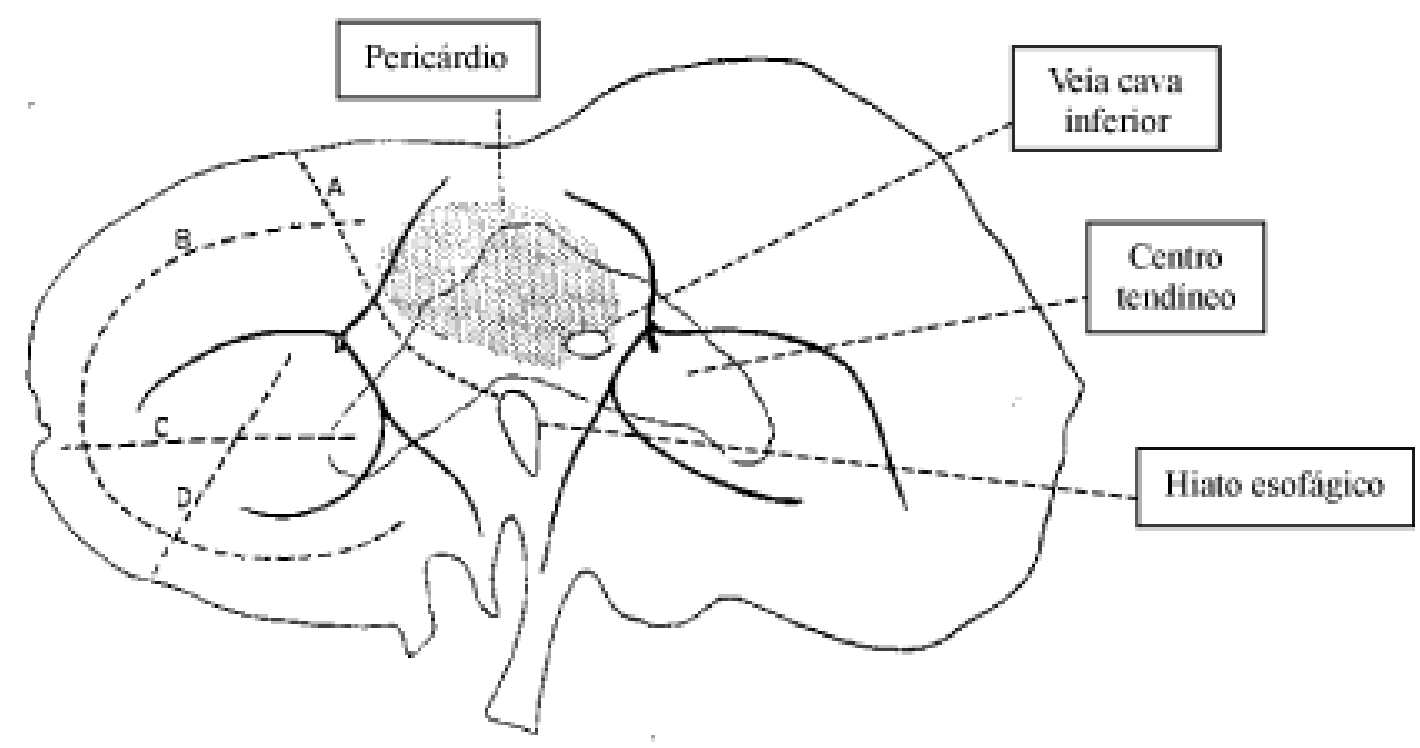

Figura 3 - Esquema da inervação frênica do diafragma mostrando os locais mais seguros para a realização de incisões no diafragma, sem o risco de efeitos significantes na função diafragmática: A, indica o componente diafragmático de incisões abdomino-torácicas combinadas, com extensão até o hiato esofágico. B, incisão circunferencial. $C$ e $D$, incisões estendendo da região costal lateral (médio-axilar) e posterior até o centro tendíneo. 


\begin{abstract}
Traumatic diaphragmatic hernia is an uncommon but important problem in the patient with multiple injuries. Since diaphragmatic injuries are difficult to diagnose, those that are missed may present with latent symptoms of bowel obstruction and strangulation. The same may occur in the patients with stab wounds to the lower chest. Traumatic diaphragmatic hernia should be suspected on the basis of an abnormal chest radiograph in the trauma victim with multiple injuries. This article discuss about history, epidemiology, clinical signs and symptoms, diagnostic modalities, treatment and complications.
\end{abstract}

Key Words: Traumatic diaphragmatic rupture, Diaphragm injury, penetrating wounds, abdominal trauma.

\section{REFERÊNCIAS}

1. Flancbaum L, Dauber M, Demas $\mathrm{C}$ et al. Early diagnosis and treatment of blunt diaphragmatic injury. Am Surg 1988; 54: 195-199.

2. Feliciano DV, Cruse PA, Mattox KL, Bitondo CG, Burch JM, Noon GP, Beall Jr AC. Delayed diagnosis of injuries to the diaphragm after penetrating wounds. J Trauma 1988; 28:1135-1144.

3. Brown GL, Richardson JD. Traumatic diaphragmatic hernia: a continuing challenge. Ann Thorac Surg 1985; 39: 170-173.

4. Uribe RA, Pachon CE, Frame SB et al. A prospective evaluation of thoracoscopy for the diagnosis of penetrating thoracoabdominal trauma. J Trauma 1994; 37: 650-654.

5. Nagy KK, Barrett JA. Diaphragm. In Rao R. Ivatury/ C. Gene Cayten: The textbook of penetrating trauma. $1^{\mathrm{a}}$ edição. Williams \& Wilkins, USA, Cap. 45, 1996; pág. 564-570.

6. Sharma OP. Traumatic diaphragmatic rupture: not na uncommon entity - Personal experience with collective review of the 1980's. J Trauma 1989; 29: 678-682.

7. Boulanger BR, Milzman DP, Rosati C et al. A comparison of right and left blunt diaphragmatic rupture. J Trauma 1993; 35:255-260.

8. Steinman M, Steinman E, Martini AC et al. Ruptura diafragmática traumática: Estudo de 35 casos. Rev Hosp Clin Fac Med S. Paulo 1993; 48:82-86.

9. Phillips T, Sclafani SJ, Goldstein A et al. Use of the contrast-enhanced CT enema in the management of penetrating trauma to the flank and back. J Trauma 1986; 26: 593-601.

10. Stylianos S, King TC. Occult diaphragm injuries at celiotomy for left chest stab wounds. Am Surg 1992; 58 : 364-368.

11. Degiannis E, Levy RD, Sofianos C et al. Diaphragmatic herniation after penetrating trauma. Br J Surg 1996; 83: 88-91.

12. Pickard LR, Mattox KL. Chest wall and diaphragm complications. In Kenneth L. Mattox: Complications of trauma, $1^{a}$ edição. Churchill Livingstone Inc., New York, 1994; Cap. 25, pág. 403-408.

13. Saber WL, Moore EE, Hopeman AR et al. Delayed presentation of traumatic diaphragmatic hernia. J Emerg Med 1986; 4: 1-7.
14. Symbas PN, Vlasis SE, Hatcher C Jr. Blunt and penetrating diaphragmatic injuries with or without herniation of organs into the chest. Ann Thorac Surg 1986; 42: 158162.

15. Shah R, Sabanathan S, Mearns AJ et al. Traumatic rupture of diaphragm. Ann Thorac Surg 1995; 60: 1444-1449.

16. Asensio JA, Demetriades D, Rodriguez A. Injury to the diaphragm. In Feliciano DV, Moore EE, Mattox KL: Trauma, $3^{\text {a }}$ edição. Appleton\&Lange. Connecticut, 1996; Cap. 29, pág. 461-485.

17. Michelson E. Eventration of the diaphragm. Surgery 1961; 49: 410-422.

18. Deslauries J. Eventration of the diaphragm. Chest Surg Clin N Am 1998; 8: 315-330.

19. Reber PU, Schmied B, Seiler CA et al. Missed diaphragmatic injuries and their long-term sequelae. J Trauma 1998; 44: 183-188.

20. Moore EE, Malangoni MA, Cogbill T et al. Organ Injury Scaling IV: thoracic, vascular, lung, cardiac and diaphragm. J Trauma 1994; 36: 299-300.

21. Mansour KA, Trauma to the diaphragm. Chest Surg Clin N Am 1997; 7: 373-383.

22. Mariadason JG, Parsa MH, Ayuyao A et al. Management of stab wounds to the thoracoabdominal region: a clinical approach. Ann Surg 1988; 207: 335-340.

23. Schackleton KL, Stewart ET, Taylor AJ. Traumatic diaphragmatic injuries: spectrum of radiographic findings. Radiographics 1998; 18: 49-59.

24. Scott JK. Diagnostic pneumoperitoneum. Br J Dis Chest 1979; 73: 55-65.

25. Shea L, Graham AD, Fletcher JC et al. Diaphragmatic injury: a method for early diagnosis. J Trauma 1982; 22: 539-543.

26. Williamson SL, Williamson MR, Golladay ES et al. Use of technetium-99m albumins colloid to assess competency of hemidiaphragms in children. Clin Nucl Med 1987; 12: 373-376.

27. Worthen NJ, Worthen WF. Disruption of the diaphragmatic echoes: a sonographic sign of diaphragmatic disease. J Clin Ultrasound 1982; 10: 43-49.

28. Demos TC, Solomon C, Posniak HV et al. Computed tomography in traumatic defects of the diaphragm. Clin Imag 1989; 13: 62-67.

29. Colliver C, Oller DW, Rose G et al. Traumatic intrapericardial diaphragmatic hernia diagnosed by echocardiography. J Trauma 1997; 42: 115-117.

30. Aldhoheyan A, Jain SK, Hamdy M et al. Traumatic intrapericardial diaphragmatic hernia. Injury 1992; 23: 331332. 
31. Serpell JW, Johnson WR. Traumatic diaphragmatic hernia presenting as na intercostal hernia: case report. J Trauma 1994; 36: 421-423.

32. Committee on Trauma, American College of Surgeons: Advanced Trauma Life Support Manual. Chicago, American College of Surgeons, 1993.

33. Merlotti GJ, Dillon BC, Lange DA et al. Peritoneal lavage in penetrating thoracoabdominal trauma. J Trauma 1988; 28: 17-23.

34. Dudley AG, Teaford H, Gatewood TS. Delayed traumatic rupture of the diaphragm in pregnancy. Obst Gyn 1979; 53(suppl): 25S-27S.

35. Bush CA, Margulies R. Traumatic diaphragmatic hernia and intestinal obstruction due to penetrating trunk wounds. South Med J 1990; 83: 1347-1350.

36. Ivatury RR, Simon RJ, Stahl WM. A critical evaluation of laparoscopy in penetrating abdominal trauma. J Trauma 1993; 34: 822-827.

37. Ochsner MG, Rozycki GS, Lucente F et al. Prospective evaluation of thoracoscopy for diagnosing diaphragmatic injury in thoracoabdominal trauma: a preliminary report. J Trauma 1993; 34: 704-709.

38. Meyers BF, McCabe CJ. Traumatic diaphragmatic hernia. Occult marker of serious injury. Ann Surg 1993; 218 : 783-790.

39. Lumsden AB, Colborn GL, Sreeran $\mathrm{S}$ et al. The surgical anatomy and technique of the thoracoabdominal incision. Surg Clin North Am 1993; 73: 633-644.

40. Feigenberg Z, Salomon J, Levy MJ. Traumatic rupture of diaphragm: surgical reconstruction with special reference to delayed closure. J Thoracic Cardiovasc Surg 1977; 74: 249-252.

41. Waldhausen JA, Kilman JW, Helman CH et al. The diagnosis and management of traumatic injuries of the diaphragm including the use of Marlex prostheses. J Trauma 1966; 6: 332-343.

42. Bender JS, Lucas CE. Management of close-range shotgun injuries to the chest by diaphragmatic transposition: case reports. J Trauma 1990; 30: 1581-1584.

43. Rasiah KK, Crowe PJ. Laparoscopy repair of a traumatic diaphragmatic hernia. J Laparoendosc Surg 1995; 5:405-407.
44. Ivatury RR, Simon RJ, Weksler B et al. Laparoscopy in the evaluation of the intrathoracic abdomen after penetrating injury. J Trauma 1992; 33: 101-108.

45. Merendino KA, Johnson RJ, Skinner HH et al. The intradiaphragmatic distribution of the phrenic nerve with particular reference to the placement of diaphragmatic incisions and Controlled Segmental Paralysis. Surgery 1956; 39: 189-198.

46. Fell SC. Surgical anatomy of the diaphragm and the phrenic nerve. Chest Surg Clin N Am 1998; 8: 281-294.

Endereço para correspondência:

Dr. Gerson Alves Pereira Júnior

Rua Iguape, 747 apto 12 - C

Jardim Paulista

CEP 14090-000

Ribeirão Preto - SP

e-mail: gersonapj@netsite.com.br 